\title{
Interactive effects of biostimulants and water stress on potted Pinot noir grapevines.
}

\author{
Sofia Sbraci $^{1,}{ }^{*}$, Linda Salvi $^{1}$, Francesca Paoli $^{1}$, Eleonora Cataldo ${ }^{1}$, Alessandra Zombardo ${ }^{2}$, Sergio Puccioni $^{2}$, Paolo \\ Storchi ${ }^{2}$ and Giovan Battista Mattii ${ }^{1}$ \\ ${ }^{1}$ DISPAA, Dipartimento di Scienze delle Produzioni Agroalimentari e dell'Ambiente, Università di Firenze, Viale delle Idee 30, 50019, \\ Sesto Fiorentino (FI), Italia \\ ${ }^{2}$ CREA-VIC, consiglio per la ricerca in agricoltura e l'analisi dell'economia agraria, Unità di Ricerca per la Viticoltura, Arezzo
}

\begin{abstract}
Seaweeds extracts (SWE) are innovative and non-polluting tools that have become popular as biostimulants. Their effects on Pinot noir water potential, gas exchanges and fruit quality under drought stress and full irrigation were evaluated during the 2017 growing season. Differential irrigations were applied and Pinot Noir grapevines have been sprayed two times at label doses $(3 \mathrm{~g} / \mathrm{L})$ during the vegetative growth, with the Ascophyllum nodosum exract, starting from veraison. During the season, measurements of single leaf gas exchange and stem water potential were made; moreover parameters of technological maturity ( ${ }^{\circ}$ Brix, acidity, $\left.\mathrm{pH}\right)$ and phenological one were analyzed. The inhibition of gas exchange and increase of stomatal limitation induced by drought stress were not reduced by SWE where A.nodosum had positive effects on midday stem water potential. Under full irrigation SWE increased leaf gas exchange. No particular effect was observed on the technological parameters after the treatment. The application of the seaweed extract improved polyphenols accumulation in stressed theses, increasing wine grapes quality. A.nodosum can be useful to reduce negative effects of abiotic stress such lack of water and to improve polyphenols content in grapes, especially in genotypes with a limited phenolic profile like Pinot Noir variety.
\end{abstract}

\section{Introduzione}

Ascophyllum nodosum è un'alga bruna il cui estratto viene riconosciuto da tempo come biostimolante per il miglioramento delle performance qualitative di diverse colture [1]. Su piante di melo il trattamento fogliare ha determinato un incremento della resa e della qualità dei frutti e, su piante di olivo, il trattamento effettuato in prefioritura ha aumentato la resa e la qualità dell'olio [2-3]. Un ulteriore interessante effetto è stato dimostrato in caso di stress abiotici. Su Spinacia oleracea L., l'applicazione di $A$. nodosum ha ridotto la chiusura stomatica e l'inibizione degli scambi gassosi, sotto condizioni di stress idrico [4]. Infine, su uve da vino ed in particolare su Pinot Nero, Cabernet Franc e Sangiovese, il trattamento medio-tardivo con $A$. nodosum, ha portato un significativo aumento del contenuto di antociani e polifenoli della bacca, mostrando i primi risultati positivi sulla qualità delle uve da vino e soprattutto su quella di varietà con genotipo a ridotto profilo polifenolico, come il Pinot Nero [5]. La presente ricerca ha l'obiettivo di valutare la risposta fisiologica e qualitativa a trattamenti con A. nodosum in viti di Pinot Nero coltivate in vaso e soggette a regimi idrici differenziati. In particolare, l'intenzione è quella di evidenziare gli effetti sulla risposta fisiologica delle piante trattate, in termini di relazioni idriche e scambi gassosi, con particolare attenzione alla valutazione delle caratteristiche qualitative delle uve, da riscontrare nei contenuti di zucchero e polifenoli delle bacche. Il principale fine sarà quello di valutare l'effetto del trattamento all'interno di uno stesso regime idrico e di una stessa fase fenologica.

\section{Materiali e metodi}

Il progetto è stato svolto presso l'Unità di ricerca per la Viticoltura del CREA di Arezzo, su Pinot Nero innestato su 1103 Paulsen (PAU). Le viti, di10 anni, sono coltivate in vasi di plastica da $75 \mathrm{~L}$ con suolo di medio impasto. Il trattamento con l'estratto $A$. nodosum di Sipcam Italia s.p.a, è stato effettuato a tutta chioma e distribuito nella quantità di $3 \mathrm{~g} / \mathrm{L}$ secondo disegno sperimentale uno schema a blocchi randomizzati; nello specifico sono stati impostati tre blocchi costituiti da 30 vasi ciascuno. Sono stati inoltre adottati regimi idrici differenziati tramite ausilio di impianto a goccia, per la delineazione delle seguenti tesi:

- WW, viti non stressate, con valori di potenziale idrico di tralcio $(\psi)$ mantenuti tra $-1,0$ e $-1,3$ $\mathrm{MPa}$ misurato a mezzogiorno solare. 
- WS, viti sottoposte a stress idrico, con valori di potenziale di tralcio mantenuti tra $-1,5$ e $-2,0$ $\mathrm{MPa}$ misurato a mezzogiorno solare.

Ai testimoni WW e WS sono stati successivamente applicati i trattamenti di biostimolante che hanno così determinato le due ulteriori tesi:

- WWt, ovvero viti ben irrigate e trattate con estratto di alga bruna $A$.nodosum

- WSt, ovvero viti stressate e trattate con lo stesso estratto.

A. nodosum è stato distribuito due volte nel corso della stagione vegetativa: in corrispondenza delle fase fenologica di invaiatura e 10 giorni prima della raccolta. Al fine di una corretta valutazione degli effetti del trattamento, sono stati individuati tre tempi di rilievi:

- rilievi al tempo zero (t0), effettuati in preinvaiatura, precedentemente all'applicazione del biostimolante;

- rilievi al tempo uno (t1), effettuati in fase di invaiatura, dopo la prima applicazione di biostimolante;

- rilievi al tempo due (t2), effettuati in fase di vendemmia, in seguito alla seconda applicazione di biostimolante.

Durante la sperimentazione sono stati monitorati i valori di potenziale idrico, mediante camera a pressione Scholander (PMS 600 EXP), e gli scambi gassosi fogliari, misurati con analizzatore di gas all'infrarosso (CIRAS 3, PP Systems Amesbury, MA USA).

Le analisi tecnologiche sulle uve, condotte in laboratorio, hanno previsto la determinazione del grado zuccherino con rifrattometro ottico ( ${ }^{\circ}$ Brix), del $\mathrm{pH}$ e dell'acidità totale del mosto $(\mathrm{g} / \mathrm{L}$ acido tartarico). Infine, le analisi fenoliche delle uve, in termini di contenuto polifenolico totale $(\mathrm{mg} / \mathrm{L})$, sono state effettuate con il metodo Yves-Glories. I dati raccolti sono stati sottoposti all'analisi della varianza e separazione delle medie tramite il test LSD con SPSS Data Editor.

\section{Risultati e discussione}

Nella valutazione degli effetti biostimolante-regime idrico su piante di Pinot nero allevate in vaso, emerge un positivo effetto del trattamento sui livelli di potenziale idrico, rispetto al controllo. Come si osserva nella Figura 1, il trattamento incide significativamente sui livelli di potenziale idrico dopo la seconda applicazione di biostimolante, mostrando un interessante potere di mitigazione degli effetti dovuti alla mancanza di acqua. Inoltre, l'analisi statistica dei dati, effettuata tra le tesi con stesso regime idrico ed all'interno della stessa fase fenologica, evidenzia un importante effetto del trattamento sulle tesi stressate al t2 (Fig 2), momento in cui i valori di potenziale della tesi WSt sono meno negativi rispetto a quelli delle tesi non trattate. Tale effetto non si registra sulle tesi ben irrigate, che comunque non raggiungono mai livelli di stress idrico severo, grazie all'effetto dell'irrigazione (Fig 3).

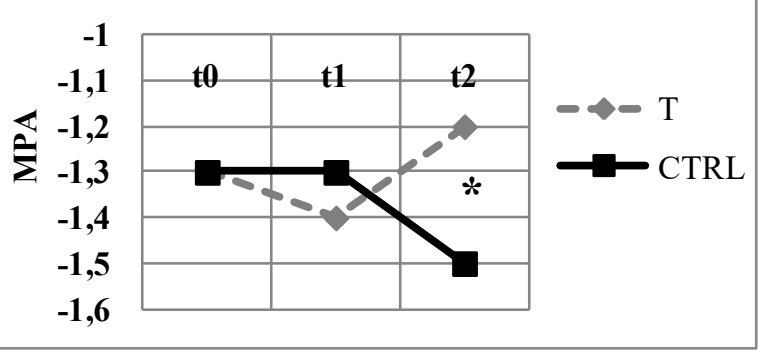

Fig. 1. Effetto del trattamento sul potenziale idrico stagionale a mezzogiorno solare. ${ }^{*}$ indica significatività $(\mathrm{P} \leq 0,05)$

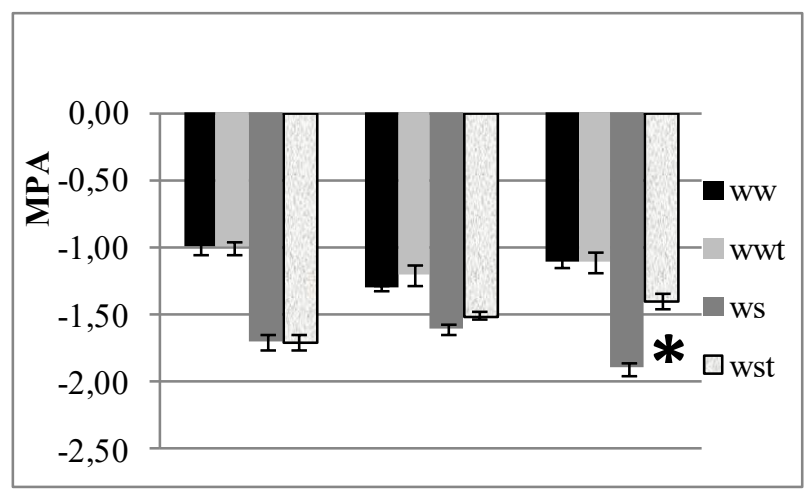

Fig. 2. Potenziale idrico nelle tre date di rilievo. I valori rappresentano la media $( \pm \mathrm{SE}, \mathrm{n}=10)$ delle misure idriche di tralcio registrate a mezzogiorno. * indica significatività $(\mathrm{P} \leq$ $0,05)$. Le differenze statistiche sono da intendersi tra le tesi all'interno dello stesso regime idrico e della stessa fase fenologica.

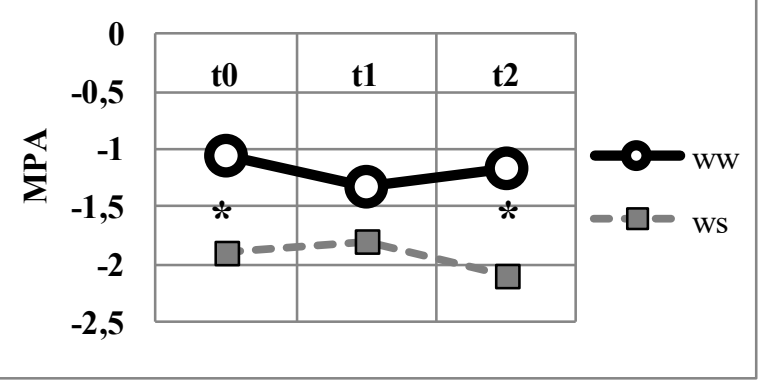

Fig 3. Effetto dell'irrigazione sul potenziale idrico stagionale a $\mathrm{m}$. * indica significatività $(\mathrm{P} \leq 0,05)$.

Per quanto riguarda gli scambi gassosi fogliari (Fig. 4,5,6), si evidenzia un aumento significativo della conduttanza stomatica (gs) e della traspirazione (E) per le tesi WWt dopo entrambe le applicazioni di biostimolante, con conseguente aumento dell'attività fotosintetica, più evidente al rilievo $t 2$ dove la differenza 
tra WW e WWt è statisticamente significativa. Per le tesi sottoposte a stress idrico sembra invece che il trattamento non abbia determinato lo stesso significativo miglioramento delle performance fisiologiche riscontrato nelle tesi ben irrigate. Infatti, nonostante il trattamento sia riuscito a rendere meno negativo il potenziale idrico delle tesi stressate, i valori di gs, Pn e traspirazione delle stesse risultano piuttosto bassi. Per spiegare questo risultato potremmo ipotizzare una tendenza isoidrica della varietà Pinot Nero. Come dimostrato per il vitigno Montepulciano [6], la varietà a carattere isoidrico, o cosiddetta "pessimista", tende a mantenere $\psi$ della foglia sopra una certa soglia, abbassando al contempo gs al ridursi della disponibilità idrica. Allo stesso modo, si può ipotizzare che lo stress idrico prolungato della stagione 2017 abbia indotto le piante di Pinot Nero a ridurre prontamente la traspirazione chiudendo gli stomi e riducendo la propria attività fotosintetica. Se ne deduce che probabilmente l'effetto del trattamento, seppur interessante, non è risultato tale da riuscire ad interferire con un meccanismo di autoregolazione di risposta al deficit idrico tipico di varietà classificate come isoidriche, di cui il Pinot Nero può rappresentare un esempio.

Nella valutazione degli effetti del trattamento sulla qualità delle uve, prendiamo in esame i valori delle analisi tecnologiche e fenoliche a vendemmia. Come riportato in Tabella 1, non si evidenziano particolari effetti del trattamento sui parametri tecnologici. In generale si registra un minore accumulo zuccherino per la tesi WW ed un peso dell'acino significativamente maggiore nella tesi WSt rispetto a WS; il trattamento ha probabilmente attenuato l'effetto di disidratazione dell'acino dovuto alla mancanza di acqua. Infine, per quanto riguarda le analisi fenoliche, da sottolineare vi è un aumento significativo dei polifenoli totali nelle tesi stressate, subito dopo il primo trattamento con $A$. nodosum (Fig. 7). La tendenza si mantiene nel corso della stagione fino a vendemmia, determinando, come evidenziato in altri lavori [5], una migliore qualità delle uve trattate. Infatti, se da una parte il contenuto di polifenoli totali nelle tesi stressate tende a diminuire nel corso della stagione, per un probabile effetto di degradazione degli stessi, dall'altra il trattamento tende ad attenuare tale fenomeno nelle tesi WSt. Per le tesi WW, il trattamento sembra non incidere sul miglioramento delle caratteristiche fenoliche ma l'effetto degradazione risulta generalmente meno evidente.

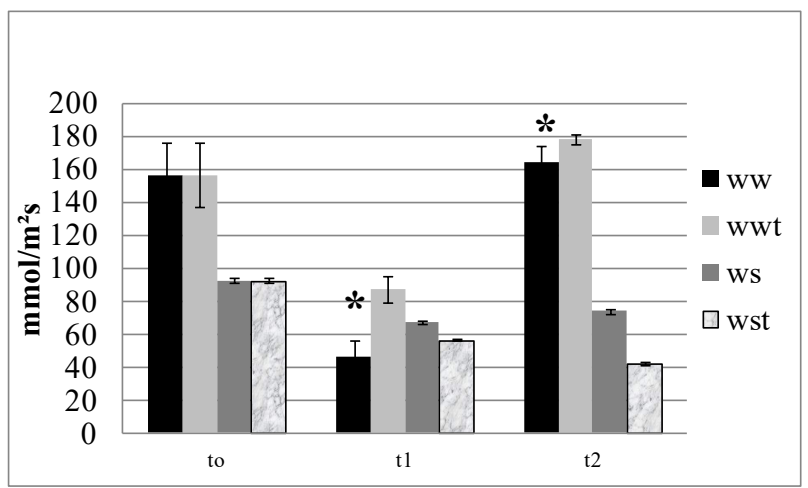

Fig. 4. Conduttanza stomatica registrata nelle tre date di rilievo. I valori rappresentano la media $( \pm S E, n=5)$ dei valori misurati su singola foglia. * indica significatività $(\mathrm{P} \leq 0,05)$.

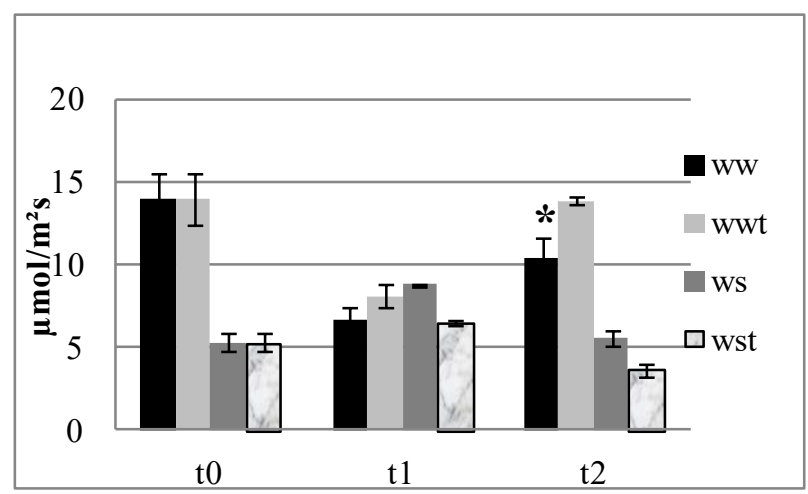

Fig. 5. Fotosintesi netta registrata nelle tre date di rilievo. I valori rappresentano la media $( \pm \mathrm{SE}, \mathrm{n}=5)$ dei valori misurati su singola foglia. * indica significatività $(\mathrm{P} \leq 0,05)$

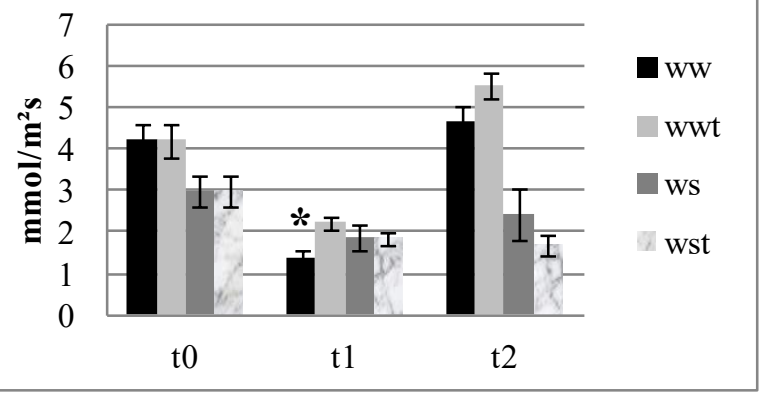

Fig. 6. Traspirazione registrata nelle tre date di rilievo. I valori rappresentano la media $( \pm \mathrm{SE}, \mathrm{n}=5)$ dei valori misurati $\mathrm{su}$ singola foglia. * indica significatività $(\mathrm{P} \leq 0,05)$

Tab. 1. Valori dei parametri tecnologici delle uve alla vendemmia.

\begin{tabular}{|c|c|c|c|c|}
\hline \multirow{2}{*}{ tesi } & Peso/acino & \multirow{}{*}{ Brix } & pH & Acidità \\
\cline { 2 - 3 } \cline { 5 - 5 } & $(\mathrm{g})$ & & & $\begin{array}{c}(\mathrm{g} / \mathrm{L} \text { ac. } \\
\text { tartarico }\end{array}$ \\
\hline WW & 1,02 & 17,6 & 3,52 & 5,37 \\
\hline WWt & 1,07 & 18,73 & 3,47 & 5,57 \\
\hline DMS & $n s$ & $n s$ & $n s$ & $n s$ \\
\hline WS & $\mathbf{0 , 8 7}$ & 19,13 & 3,5 & 5,57 \\
\hline WSt & 1,02 & 19,33 & 3,48 & 5,93 \\
\hline DMS & $\mathbf{0 , 0 9 2}$ & $n s$ & $n s$ & $n s$ \\
\hline
\end{tabular}




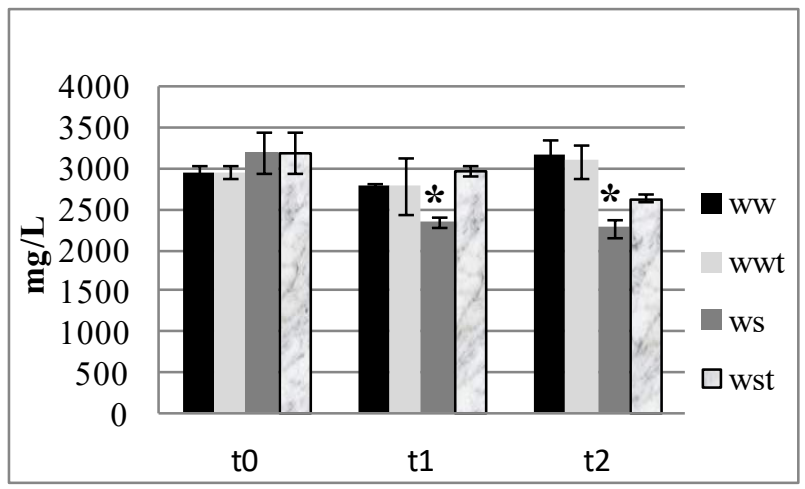

Fig. 7. Contenuto di polifenoli totali nelle tre date di rilevo. I valori rappresentano la media $( \pm \mathrm{SE}, \mathrm{n}=10)$ dei valori ottenuti nei campioni analizzati. * indica significatività $(\mathrm{P} \leq 0,05)$

\section{Conclusioni}

L'estratto di Ascophyllum nodosum rappresenta un innovativo e sostenibile prodotto biostimolante da utilizzare in agricoltura per il miglioramento delle performance qualitative delle colture. Il suo utilizzo in campo viticolo e soprattutto su uve da vino è in continua sperimentazione, ma ha fornito fino ad oggi importanti risultati sulla risposta fisiologica della pianta e sulla qualità delle uve. L'applicazione del biostimolante in regimi idrici differenziati è risultato interessante per valutarne gli effetti in caso di stress idrico, condizione sempre più comune nelle aree viticole sottoposte al cambiamento climatico degli ultimi decenni. L'applicazione del prodotto in fasi vegetative mediotardive ha portato ad un'attenuazione generale dei fenomeni di stress idrico a cui le piante erano sottoposte. Inoltre, l'esperimento ha evidenziato un importante effetto sulle caratteristiche qualitative delle uve ed in particolare sul contenuto di polifenoli della bacca di uve stressate e maggiormente soggette a fenomeni di degradazione dei composti fenolici. Questo effetto risulta interessante per quelle varietà, come il Pinot Nero, a ridotto profilo polifenolico. Altri approfondimenti saranno necessari per comprendere al meglio il reale meccanismo di azione dell'estratto di alga bruna ed in particolare il suo contributo nella regolazione dei processi metabolici delle piante.

\section{Bibliografia}

1. D. Battacharyya, M.Z. Babgohari, P. Rathor, B. Prithiviraj, Sci. Hortic. 196, 39-48 (2015)

2. A. Basak, international Journal of Fruit Science, 8, 1-14 (2008)

3. V. Chouliaras, M. Tasioula, C. Christos, I. Therios, E.Tsabolatidou. Sci Food Agr 89(6), 984-988 (2009)

4. Daniel. I. Leskovar, C. Xu, Scientia Horticulturae 183, 39-47 (2015)

5. T. Frioni, P. Sabbatini, S. Tombesi, J. Norrie, S. Poni, M. Gatti, A. Palliotti, 232, 97-106 (2018)

6. S. Tombesi, S. Poni, A. Palliotti, Italus Hortus 23(1), 45-53 (2016) 